Психология. Журнал Высшей школы экономики.

2021. T. 18. № 1. C. 211-223. DOI: 10.17323/1813-8918-2021-1-211-223

\title{
ЭФФЕКТ ВЗГЛЯДА-ПОДСКАЗКИ В ВИРТУАЛЬНОЙ СРЕДЕ: ВЛИЯНИЕ СОЦИАЛЬНОЙ УСТАНОВКИ И СОЦИАЛЬНОЙ ДИСТАНЦИИ
}

\author{
А.О. ПЕТРОВА , Е.Г. ЛУНЯКОВА \\ ${ }^{a}$ Московский государственньй университет имени М.В. Ломоносова, 119991, Россия, Москва, \\ Ленинские горы, Ә. 1 \\ ${ }^{b}$ Национальньй исследовательский университет «Высшая школа экономики», 101000, Россия, \\ Москва, ул. Мясницкая, д. 20 \\ ' Российская академия народного хозяйства и государственной службы при Президенте \\ Российской Федеращии, 119571, Россия, Москва, просп. Вернадского, д. 82, стр. 1
}

\begin{abstract}
Резюме
Пространственное внимание людей, вовлеченных в совместную деятельность, часто направлено на одни и те же объекты среды. При этом перенаправление взгляда одного из партнеров становится средством управления вниманием других. Можно предположить, что на выраженность активно исследуемого в последние десятилетия эффекта взгляда-подсказки будут оказывать влияние такие социально-психологические факторы, как установка в отношении подсказывающего и социальная дистанция между испытуемым и подсказывающим. Для проверки этих предположений была создана виртуальная 3D-среда, в которой был реализован модифицированный вариант методики подсказки М. Познера с соавт. (Posner et al., 1978). Использовался межгрупповой экспериментальный план. Для одной группы испытуемых антропоморфный аватар, дающий подсказку взглядом, выступал, согласно предварительной инструкции, в роли помощника, для другой - в роли человека, оценивающего действия испытуемого. Аватар мог давать верные и неверные подсказки взглядом относительно места появления целевого объекта. Обе группы проходили по две экспериментальные серии, различавшиеся дистанцией между испытуемым и аватаром: 1.5 м (комфортная дистанция, соответствующая зоне формальных социальных контактов) и 1 м (некомфортная дистанция, соответствующая зоне личных контактов). Эффект взгляда-подсказки был выявлен во всех экспериментальных условиях в виртуальной среде. При этом он был сильнее выражен в условиях «помогающей» установки, чем в условиях «оценивающей». В условиях помогающей установки эффект был несимметричен: выигрыш при верной подсказке оказался меньше, чем задержка реакции на стимул при неверной. Для обеих установок эффект взгляда-подсказки сильнее проявлялся при дистанции 1 м до аватара, чем при дистанции 1.5 м. Последнее может быть связано как с большими угловыми размерами подсказки, так и с размыванием границ личной зоны в виртуальной среде. Результаты исследования могут быть использованы при создании обучающих виртуальных сред.
\end{abstract}

Ключевые слова: эффект взгляда-подсказки, пространственное внимание, виртуальная реальность, социальная установка, социальная дистанция.

Участие М.В. Фаликман поддержано грантом РФФИ и Фонда «За русский язык и культуру» (РЯИК) в Венгрии № 20-511-23001. 
Все больше внимания в современной когнитивной психологии уделяется изучению влияния мотивационных, эмоциональных и социальных факторов на когнитивные процессы человека. Например, важную роль в управлении пространственным вниманием играет взгляд другого человека, что выражается в активно изучаемом в последние два десятилетия эффекте взгляда-подсказки (Friesen, Kingstone, 1998). Однако накоплено недостаточно данных о том, насколько этот эффект подвержен влиянию социальных факторов, таких как установка в отношении подсказывающего и межличностная дистанция.

Наиболее распространенной методикой исследования пространственного внимания является методика подсказки, которую разработали М. Познер с коллегами (Posner, 1980; Posner et al., 1978). Методика нацелена на изучение «скрытой» ориентировки внимания, не связанной с движениями глаз.

Согласно результатам исследований Познера, верная центральная подсказка-стрелка, указывающая в направлении целевого стимула, ускоряет двигательный ответ на его появление, а неверная - замедляет, но только тогда, когда количество верных подсказок существенно превышает количество неверных. Если же частота предъявления верных и неверных подсказок одинакова, эффект подсказки не проявляется. Однако в последующих исследованиях при использовании в качестве центральной подсказки схематического или фотографического изображения лица человека со взглядом, указывающим вправо или влево (Driver at al., 1999; Kingstone et al., 2003), в случае правильной подсказки вне зависимости от частоты ее предъявления время реакции испытуемого снижалось. В этом плане подсказка взглядом сродни ориентировке внимания на периферические подсказки, дающие выигрыш в случае верной подсказки без проигрыша при неверной (Jonides, 1981).

Эффект более быстрого перенаправления пространственного внимания в соответствии с направлением взгляда другого человека получил название эффекта взгляда-подсказки (gaze-cueing effect) (Friesen, Kingstone, 1998; Kuhn, Kingstone, 2009).

Было установлено, что время реакции в случае с верной подсказкой значимо меньше по сравнению с временем реакции для проб с нейтральной или неверной подсказками. Этот эффект возникает относительно быстро на коротких временны́х интервалах (от 105 мс) и исчезает на более длинных временны́х интервалах.

Дальнейшие исследования показали, что на эффект взгляда-подсказки могут оказывать влияние различные социальные факторы. Среди этих факторов - политические взгляды испытуемого (Dodd et al., 2011), знакомость лиц людей, дающих подсказку взглядом (Deaner et al., 2007), социальный статус этих людей (McDonald, Tatler, 2013), надежность, приписываемая им испытуемыми и модулируемая уровнем тревожности испытуемых (Süßenbach, Schönbrodt, 2014).

Нам представляется, что одним из важных факторов, который может оказывать влияние на выраженность эффекта взгляда-подсказки, является установка испытуемого в отношении подсказывающего: в частности, воспринимается ли он испытуемым как помощник в решении экспериментальной задачи 
или как человек, выносящий оценочное суждение относительно его возможностей. Мы предполагаем, что помогающая установка вызовет больше доверия к виртуальному персонажу у испытуемого, а потому он будет больше ориентироваться на подсказку, что положительно повлияет на выраженность эффекта взгляда-подсказки.

В свою очередь, влияние установки в отношении подсказывающего может модулироваться таким фактором межличностного взаимодействия, как социальная дистанция. В межличностном общении Э. Холл (Hall, 1968) определяет четыре зоны: интимная $(<0.45$ м), личная $(0.45-1.2$ м), социальная (от 1.2 до 3.6 м) и общедоступная (> 3.6 м). С незнакомыми людьми в формальных контактах человек предпочитает общаться, удерживая партнера в зоне социальной дистанции. Допуск в личную зону больше характерен для неформального и дружеского общения, присутствие в этой зоне незнакомого человека может вызывать дискомфорт. Для изучения влияния социальной дистанции и установки на эффект взгляда-подсказки возможно применение технологии виртуальной реальности.

Современные исследования показывают, что восприятие участниками человеческих аватаров, «виртуальных партнеров по общению», существенно не отличается от восприятия реальных людей. 3D-модели человека вызывают у испытуемых сопоставимые физиологические, поведенческие и нейробиологические реакции, как если бы они взаимодействовали с людьми в реальных условиях (Borst, Gelder, 2015). Это сходство в восприятии не ограничивается внешним видом виртуальных персонажей, оно также распространяется на восприятие эмоциональных выражений лица и тела (McDonnell et al., 2008).

В некоторых исследованиях (Peters et al., 2018; Zibrek et al., 2017) выявлено сходство реакций испытуемых на разные типы межличностной дистанции при взаимодействии с реальными людьми и с аватарами в виртуальной среде. Это в целом соответствует гипотезе, согласно которой социальные расстояния внутри смешанной реальности с виртуальными персонажами сопоставимы с теми, которые были найдены для межличностных расстояний человек-человек (Lambert, 2004).

В нашем исследовании мы проверяли гипотезы о том, что тип установки, заданный инструкцией, и расстояние между испытуемым и аватаром, дающим подсказку, влияют на выраженность эффекта взгляда-подсказки в виртуальной среде.

1) При «помогающей» социальной установке эффект взгляда-подсказки будет выражен сильнее, чем при «оценивающей» установке.

2) При более комфортной социальной дистанции между испытуемым и аватаром эффект будет выражен больше, чем при некомфортной.

\section{Методика}

Испытуемые: 46 человек (24 женщины и 22 мужчины) в возрасте от 18 до 28 лет с нормальным или скорректированным до нормального зрением. 
Для исследования эффекта подсказки в различных условиях была создана экспериментальная виртуальная 3D-среда. В ней была реализована авторская модификация методики М. Познера (Posner et al., 1978) с подсказкой взглядом, по которой испытуемые должны были как можно быстрее ответить на появление сигнала справа или слева от аватара нажатием на кнопку.

\section{Annapamypa}

Для предъявления стимулов в 3D-среде использовался шлем виртуальной реальности HTC Vive, угол обзора $110^{\circ}$, разрешение $2160 \times 1200$, разрешение дисплеев для каждого глаза 1200 х 1080, частота обновления 90 Гц. Ответы испытуемых регистрировались при помощи двух беспроводных совместимых со шлемом виртуальной реальности контроллеров.

Для программирования 3D-среды в эксперименте была использована интегрированная среда разработки Unity.

В качестве стимулов выступали виртуальные объекты в виде двух одинаковых серых шаров (диаметр 0.05 м), подвешенных на веревках для обеспечения более точного субъективного определения испытуемыми расстояния до них. Шары находились слева и справа от центральной точки напротив испытуемого, на угловом расстоянии 7 градусов, на расстоянии 70 см.

В роли подсказки выступало направление взгляда аватара - 3D-модели человека в полный рост мужского пола, нормального телосложения, рост подстраивался под рост испытуемого (от 140 до 210 см) так, чтобы глаза аватара находились на линии взгляда испытуемого. Средние черты лица, выразительные глаза. Анимация покоя, без саккад. Во время предъявления фиксационной точки анимация отсутствовала (см. рисунок 1 ).

Аватар располагался напротив испытуемого на платформе, расчерченной сеткой больших квадратов для верной оценки расстояния до аватара. Окружающее пространство - темно-серое.

Рисунок 1

Виртуальная среда для эксперимента: аватар-подсказка, объекты

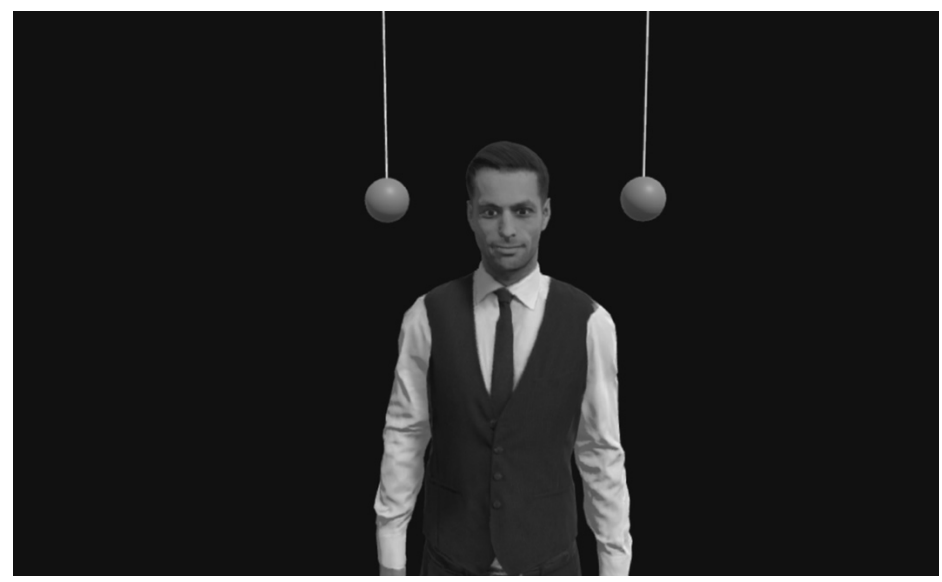


Воспринимаемое расстояние между испытуемым и аватаром в разных экспериментальных условиях составляло 1.5 м или 1 м - согласно предыдущим исследованиям (Hall, 1968; Peters et al., 2018), «комфортное» и «некомфортное».

\section{Процедура эксперимента}

Использован межгрупповой экспериментальный план. Испытуемые поделены на две группы (23 человека в каждой), для каждой группы дается одна из двух инструкций, которая предполагает разную установку по отношению к аватару-подсказке (помощник или оценщик). Перед испытуемым ставилась задача сфокусироваться на фиксационной точке и как можно быстрее нажать на правую или левую кнопку контроллера, когда справа или слева вспыхнет шар. Испытуемым одной группы сообщалось, что исследователь участвует в разработке системы для поддержки внимания при поиске объектов на экране компьютера и что перед ними находится аватар-помощник, который постарается облегчить решение задачи, но иногда может ошибаться и давать неверную подсказку. Испытуемым другой группы говорилось, что исследователь участвует в разработке системы для оценки переключаемости внимания в рамках батареи методик для подбора и оценки персонала и что аватар будет оценивать, насколько быстро и правильно испытуемый решил задачу, указывая взглядом, где загорится шар, и иногда давая неверную подсказку.

Для каждой группы эксперимент состоял из двух этапов по 200 проб в каждом, из них 100 - верные подсказки, 50 - неверные, 50 - нейтральные (аватар не переводил взгляд). Этапы различались расположением аватара-подсказки - на комфортной социальной дистанции от испытуемого $(1.5$ м) и на некомфортной $(1 \mathrm{~m})$. Испытуемые проходили этапы в разном порядке, перерыв между ними составлял 5 минут, общая продолжительность эксперимента составляла в среднем 20 минут.

Эксперимент начинался с тренировочной серии из 6 проб для ознакомления с процедурой.

Перед началом каждой пробы (см. схему на рисунке 2) в центре (на переносице аватара) предъявлялась фиксационная точка в течение 1000/2000 мс. В качестве подсказки выступал взгляд аватара. Подсказка могла быть верной (в том направлении, где затем появлялся целевой стимул), неверной (в противоположном направлении) и нейтральной

Регистрировались время реакции испытуемого на стимул и правильность ответа.

\section{Результаты}

\section{Обработка результатов}

Сравнивалось время реакции испытуемого на целевой стимул в условиях с верной и неверной подсказкой, а также ее отсутствия при разной дистанции 


\section{Схема экспериментальной пробы}

Рисунок 2

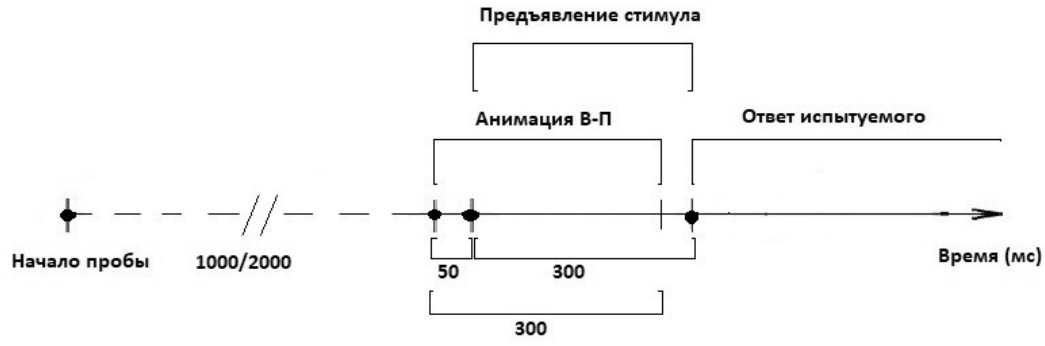

между испытуемым и аватаром и при разной установке («помогающей» и «оценивающей»). Данные обрабатывались с помощью статистических пакетов SPSS 23.0 и Jamovi 1.1. Использовались критерий Манна-Уитни для независимых выборок и многофакторный дисперсионный анализ с повторными измерениями с поправкой на сферичность Гринхауса-Гейсера и поправкой Бонферрони на множественные сравнения.

Перед проведением анализа были исключены данные для случаев, когда испытуемый делал ошибку в локализации стимула (право/лево) либо не давал ответа вообще. Данные были усреднены по каждому случаю для каждого испытуемого, так как выборка неоднородна по количеству проб для разного типа подсказок.

\section{Описание результатов}

Результаты дисперсионного анализа приведены в таблице 1.

Был получен эффект взгляда-подсказки для всех условий: при правильной подсказке скорость поиска уменьшалась, а при неправильной - увеличивалась в сравнении с нейтральной подсказкой.

Многофакторный анализ с повторными измерениями не выявил эффекта установки $(p=0.550)$, однако выявил его значимое взаимодействие с фактором подсказки.

Таблица 1

Влияние и взаимодействие факторов «Подсказка», «Расстояние», «Установка»

\begin{tabular}{|l|c|c|c|c|}
\hline \multicolumn{1}{|c|}{ Факторы/Взаимодействие факторов } & $\mathbf{F}$ & $\mathbf{d f}$ & $\boldsymbol{p}$ & $\boldsymbol{\eta}_{\mathbf{p}}$ \\
\hline Подсказка & 531.12 & 2.88 & 0.001 & 0.923 \\
\hline Расстояние & 6.07 & 1.44 & 0.018 & 0.121 \\
\hline Установка & 0.36 & 1.44 & 0.550 & 0.008 \\
\hline Подсказка $\times$ Установка & 38.02 & 1.44 & $<0.001$ & 0.464 \\
\hline Расстояние $\times$ Установка & 0.25 & 1.44 & 0.618 & 0.006 \\
\hline Подсказка $\times$ Расстояние & 39.71 & 1.88 & $<0.001$ & 0.474 \\
\hline Подсказка $\times$ Расстояние $\times$ Установка & 0.43 & 2.88 & 0.601 & 0.010 \\
\hline
\end{tabular}


Обнаружены значимые различия по фактору расстояния ( $p=0.018)$, а также значимое взаимодействие фактора подсказки и фактора расстояния для всех случаев.

Сравнение выборочных средних по критерию Манна-Уитни показало, что эффект взгляда-подсказки выражен больше в случае с «помогающей» установкой, чем в случае с «оценивающей» установкой (см. рисунки 3,4$)$.

Также эффект выражен больше при дистанции 1 м, которая была обозначена нами как «некомфортная», чем при «комфортной» дистанции 1.5 м (см. рисунки 5, 6).

Взаимодействия между фактором установки и фактором расстояния не выявлено.

Рисунок 3

Среднее время реакции для разных типов установок при расстоянии 1.5 м (мс).

Вертикальные штрихи соответствуют стандартному отклонению

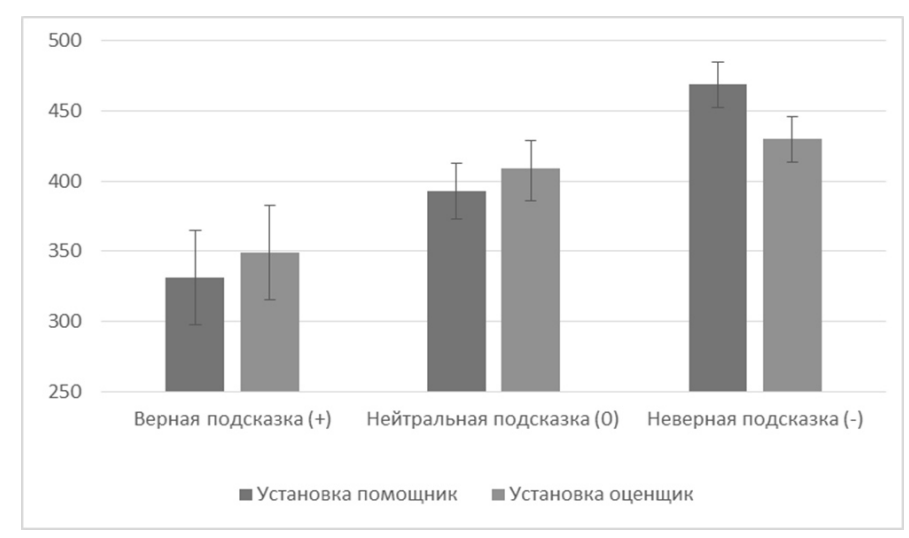

Рисунок 4

Среднее время реакции для разных типов установок при расстоянии 1 м (мс).

Вертикальные штрихи соответствуют стандартному отклонению

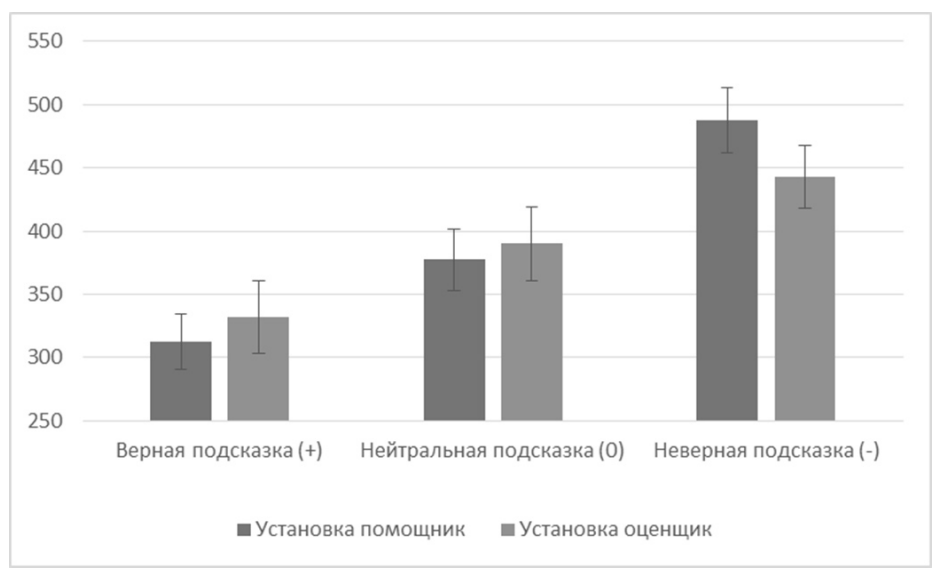


Рисунок 5

Среднее время реакции при разных расстояниях при установке «помощник» (мс). Вертикальные штрихи соответствуют стандартному отклонению

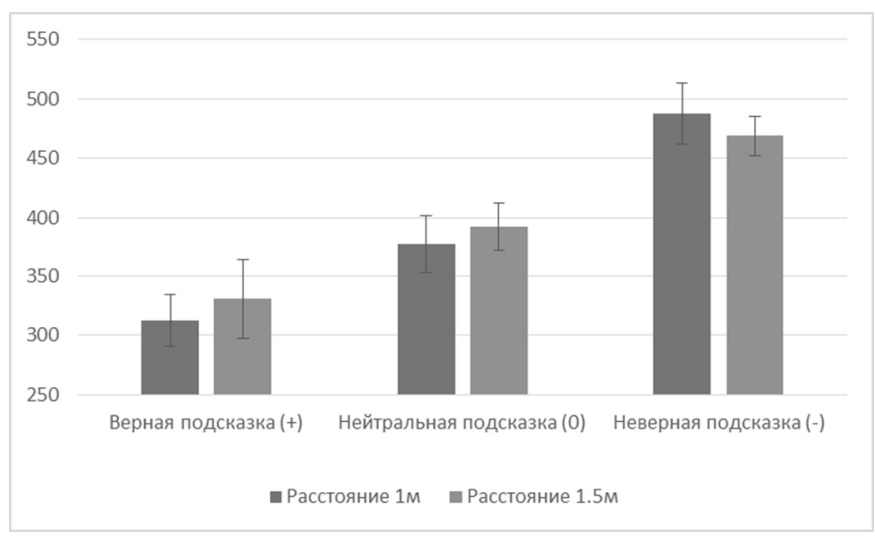

Рисунок 6

Среднее время реакции при разных расстояниях при установке «оценщик» (мс).

Вертикальные штрихи соответствуют стандартному отклонению

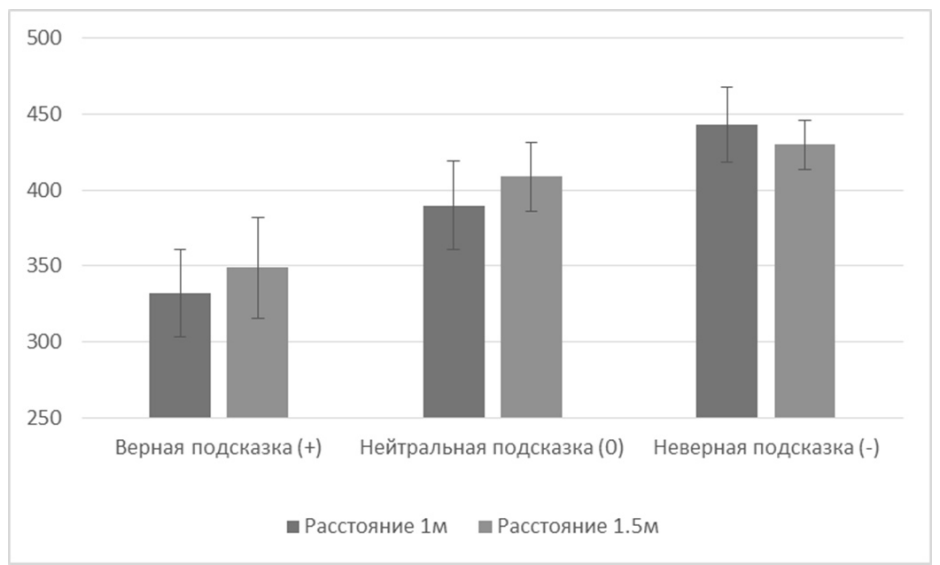

\section{Обсуждение результатов}

При верной или нейтральной подсказке в условии помогающей установки время реакции испытуемого в среднем меньше, чем при оценивающей установке. Возможно, это ускорение ответа связано с тем, что в ситуации отсутствия внешней оценки человек испытывает меньшее напряжение, связанное со страхом совершить ошибку. В случае с неверной подсказкой время реакции испытуемого, наоборот, возрастает при помогающей установке. Мы объясняем это тем, что испытуемый больше доверяет «помогающей» подсказке, 
следовательно, его больше сбивает неверная подсказка, и ему требуется больше времени, чтобы локализовать стимул.

Таким образом, в целом эффект взгляда-подсказки выражен сильнее при помогающей установке, чем при оценивающей установке. Вероятно, при оценивающей установке испытуемый имплицитно настроен на то, что ему могут дать неверные подсказки, а потому в случае с неверной подсказкой его внимание быстрее возвращается к верному местоположению стимула. И напротив, доверие к подсказке при помогающей установке дает сильно выраженный отрицательный эффект неверной подсказки, намного более существенный, чем эффект верной подсказки, тогда как в классическом эксперименте Познера эффекты верной и неверной подсказки примерно одинаковы (Posner et al., 1978). Также в исследовании К. Фризена и А. Кингстоуна со взглядомподсказкой (Friesen, Kingstone, 1998) не было обнаружено значимых различий между нейтральной и неверной подсказкой. Нельзя исключить, что наши данные могут отличаться в силу того, что в эксперименте 1998 г. были использованы схематичные изображения человеческих лиц, а в нашем - реалистичная 3D-модель человека.

Мы можем предположить, что помогающая установка - более социально желательная, чем оценивающая, что располагает к большему доверию и, следовательно, дает больший эффект подсказки. Это согласуется с результатами других исследований, в которых социально желательные черты воспринимаемого человека увеличивают склонность следовать направлению его взгляда (Deaner et al., 2007; Pavan et al., 2011; Süßenbach, Schönbrodt, 2014). Однако не все так однозначно: в двухэтапном эксперименте 2017 г. (Carraro et al., 2017) были получены противоположные результаты. На этапе обучения разные лица систематически ассоциировались с поощряемым или порицаемым поведением, после чего они были использованы в качестве стимулов в задаче со взглядом-подсказкой. Обнаружилось, что лица, связанные с антисоциальным поведением, нарушающим нормы, вызывают более сильные эффекты подсказки, чем лица, связанные с социально поощряемым поведением. Авторы исследования связывают это с тем, что взгляд людей, которым приписывается девиантное поведение, может быть сигналом, позволяющим лучше контролировать потенциально опасную ситуацию. Тем не менее мы можем предположить, что при оценивающей установке позиция аватара не воспринималась испытуемыми как социально нежелательная, в то же время помогающая установка имела больший эффект в силу того, что провоцировала испытуемых больше доверять аватару.

Противоречит нашему исходному предположению то, что эффект подсказки выражен больше при дистанции 1 м, которую мы рассматривали как «некомфортную», чем при «комфортной» дистанции 1.5 м, вне зависимости от того, какая установка была задана инструкцией. Полученный результат может объясняться большей субъективной удаленностью от испытуемого, а следовательно, и качеством опознания объекта. Кроме того, при отдалении аватара на расстояние в 1.5 м происходило уменьшение угловых размеров подсказки. Также можно предположить, что в ситуации коммуникации с виртуальным 
партнером восприятие испытуемым личных границ может размываться, в том числе в зависимости от типа решаемой задачи. Наконец, при разработке процедуры эксперимента не было предусмотрено никаких дополнительных тестов для субъективной оценки испытуемыми комфортности расстояния до аватара. Все это следует учесть при дальнейшем исследовании социальной дистанции в виртуальной реальности.

Таким образом, в нашей работе впервые получен эффект взгляда-подсказки в виртуальной среде, а также установлено, что выраженность этого эффекта меняется в зависимости от установки испытуемого в отношении аватара, дающего подсказку.

Результаты исследования могут быть использованы при создании обучающих виртуальных сред. Одним из основных элементов обучающей виртуальной среды является аватар-тьютор, в задачи которого входит максимально эффективное переключение внимания участников между элементами среды с целью более продуктивного обучения. Вопрос об относительном пространственном расположении участника обучения и аватара также важен в создании обучающих сред, поскольку тоже может оказывать влияние на эффективность переключения внимания, однако данный вопрос нуждается в дальнейшем уточнении.

Ссылки на зарубежные источники см. в разделе References после англоязычного блока.

Петрова Анастасия Олеговна - выпускник, факультет психологии, МГУ имени М.В. Ломоносова.

Сфера научных интересов: виртуальная реальность, зрительное внимание, психология личности.

Контакты: anastasiyap2305@yandex.ru

Лунякова Елизавета Геннадьевна - старший научный сотрудник, лаборатория «Восприятие», факультет психологии, МГУ имени М.В. Ломоносова, кандидат психологических наук.

Сфера научных интересов: психология восприятия, движения глаз в перцептивных процессах.

Контакты: eglun@mail.ru

Фаликман Мария Вячеславовна - руководитель, профессор, департамент психологии, факультет социальных наук; ведущий научный сотрудник, лаборатория когнитивных исследований, НИУ ВШЭ; научный сотрудник, ИОН РАНХиГС, доктор психологических наук.

Сфера научных интересов: зрительное внимание, перцептивные задачи, нисходящие влияния на обработку зрительной информации.

Контакты: mfalikman@hse.ru 


\title{
Gaze Cueing in Virtual Reality: Effects of Social Set and Social Distance
}

\author{
A.O. Petrova ${ }^{\mathrm{a}}$, E.G. Luniakova ${ }^{\mathrm{a}}$, M.V. Falikman ${ }^{\mathrm{b}, \mathrm{c}}$ \\ ${ }^{a}$ Lomonosov Moscow State University, 1 Leninskie Gory, Moscow, 119991, Russian Federation \\ ${ }^{b}$ HSE University, 20 Myasnitskaya Str., Moscow, 101000, Russian Federation \\ ${ }^{c}$ Russian Presidential Academy of National Economy and Public Administration (The Presidential \\ Academy, RANEPA), 82 build. 1, Prospect Vernadskogo, Moscow, 119571, Russian Federation
}

\begin{abstract}
Spatial attention of people involved in a co-operative activity is often directed to the same objects in the environment. At the same time, one of the partners' gaze redirection can be involved in controlling attention of others. It could be assumed that the degree of the gaze cueing effect, which has become a focus of growing research interest in recent decades, might be influenced by such social psychological factors as one's attitude towards the partner and social distance between the partners. To test these assumptions, a virtual 3D environment was created, in which a modified version of the cueing paradigm by Posner et al. (1978) was implemented. An intergroup experimental design was used. For one group of participants, the anthropomorphic avatar was introduced in the instruction as a "virtual assistant", for the other group, it was presented as a "virtual assessment specialist". The avatar could provide valid and invalid gaze cues regarding the future target location. Both groups participated in two experimental sessions, in one session the distance between the participant and the avatar was $1.5 \mathrm{~m}$ (comfortable distance corresponding to the zone of formal social contacts) and in the other session the distance was 1 $\mathrm{m}$ (uncomfortable distance corresponding to the zone of personal contacts). The gaze cueing effect was observed through all experimental conditions in a virtual environment. However, it was more pronounced for the "assisting" set than for the "assessing" one. Interestingly, for the assisting set, the effect was asymmetric: the gain due to a valid cue turned out to be less pronounced than the delay in the response to a target after an invalid cue. For both conditions, the gaze cueing effect was more pronounced at a distance of $1 \mathrm{~m}$ between the participant and the avatar than at a distance of $1.5 \mathrm{~m}$. The latter result could be associated either with the large angular dimensions of a gaze cue or with blurring of boundaries of the personal zone in the virtual environment. The results can be applied in the development of educational virtual environments.
\end{abstract}

Keywords: Gaze cueing effect, spatial attention, virtual reality, social set, social distance.

\section{References}

Borst, A. W., \& Gelder, B. D. (2015). Is it the real deal? Perception of virtual characters versus humans: An affective cognitive neuroscience perspective. Frontiers in Psychology, 6, 576.

Carraro, L., Dalmaso, M., \& Castelli, L. (2017). The appeal of the devil's eye: social evaluation affects social attention. Cognitive Processing, 18(1), 97-103. 
Deaner, R. O., Shepherd, S. V., \& Platt, M. L. (2007). Familiarity accentuates gaze cueing in women but not men. Biological Letter, 3, 64-67.

Dodd, M. D., Hibbing, J. R., \& Smith, K. B. (2011). The politics of attention: gaze-cuing effects are moderated by political temperament. Perception, 73(1), 24-29.

Driver, J., Davis, G., Ricciardelli, P., Kidd, P., Maxwell, E., \& Baron-Cohen, S. (1999). Gaze perception triggers reflexive visuospatial orienting. Visual Cognition, 6, 509-540.

Friesen, C. K., \& Kingstone, A. (1998). The eyes have it! Reflexive orienting is triggered by nonpredictive gaze. Psychonomic Bulletin and Revier, 5, 490-495.

Hall, E. T. (1968). Proxemics. Current Anthropology, 2(9). 83-108.

Jonides, J. (1981). Voluntary versus automatic control over the mind's eye's movement. In J. B. Long \& A. D. Baddeley (Eds.), Attention and performance IX (pp. 187-203). Hillsdale, NJ: Erlbaum.

Kingstone, A., Smilek, D., \& Ristic, J. (2003). Attention, researchers! It is time to take a look at the real world. Current Directions in Psychological Science, 12, 176-180.

Kuhn, G., \& Kingstone, A. (2009). Look away! Eyes and arrows engage oculomotor responses automatically. Attention, Perception and Psychophysics, 71, 314-327.

Lambert, D. (2004). Body language. London, UK: Harper Collins.

McDonald, R. G., \& Tatler, B. W. (2013). The effect of social roles on gaze cue utilization in a realworld collaboration. In Proceedings of 35th Annual Cognitive Science Society Meeting (CogSci 2013) (pp. 942-947). Austin, TX: Cognitive Science Society.

McDonnell, R., Jorg, S., \& McHugh, J. (2008). Evaluating the emotional content of human motions on real and virtual characters. In Proceedings of the 5th Symposium on Applied Perception in Graphics and Visualization APGV, Los Angeles, CA, USA, 9-10 August 2008 (pp. 67-74). New York, NY: ACM Press.

Pavan, G., Dalmaso, M., \& Galfano, G. (2011). Racial group membership is associated to gaze-mediated orienting in Italy. Journal of Intellectual Disability Research, 14, 317-326.

Peters, C., Li, C., Yang, F., Avramova, V., \& Skantze, G. (2018). Investigating social distances between humans, virtual humans and virtual robots in mixed reality. In 17th International Conference on Autonomous Agents and Multiagent Systems (AAMAS 2018) (p. 437). Richland, SC: International Foundation for Autonomous Agents and Multiagent Systems.

Posner, M. I. (1980). Orienting of attention. Quarterly Journal of Experimental Psychology, 32, 3-25.

Posner, M. I., Nissen, M. J., \& Ogden, W. C. (1978). Attended and unattended processing modes: The role of set for spatial location. In H. L. Pick \& I. J. Saltzman (Eds.), Modes of perceiving and processing information (pp. 160-174). Hillsdale, NJ: Lawrence Erlbaum Associates.

Süßenbach, F., \& Schönbrodt, F. (2014). Not afraid to trust you: Trustworthiness moderates gaze cueing but not in highly anxious participants. Journal of Cognitive Psychology, 26, 670-678.

Zibrek, K., Kokkinara, E., \& McDonnell, R. (2017). Don't stand so close to me: investigating the effect of control on the appeal of virtual humans using immersion and a proximity-based behavioral task. In Proceedings of the ACM Symposium on Applied Perception (SAP '17) (Vol 41, art. 3, p. 1-11). New York, NY: ACM.

Anastasia O. Petrova - Graduate, Faculty of Psychology, Lomonosov Moscow State University. Research Area: virtual reality, visual attention, personality psychology.

E-mail: anastasiyap2305@yandex.ru 
Elizaveta G. Luniakova - Senior Research Fellow, Laboratory of Perception, Faculty of Psychology, Lomonosov Moscow State University, PhD in Psychology.

Research Area: psychology of perception, eye movements and perception.

E-mail: eglun@mail.ru

Maria V. Falikman - Head, Professor, School of Psychology; Lead Research Fellow, Cognitive Research Laboratory, HSE University; Research Fellow, Institute for Social Sciences, RANEPA, PhD in Psychology, Dr.habil.

Research Area: visual attention, perceptual tasks, top-down influences upon visual information processing.

E-mail: mfalikman@hse.ru 